\title{
NUMBER OF COLLISIONS IN THE GLAUBER MODEL AND BEYOND
}

\author{
C. Ciofi degli Atti, ${ }^{1}$ B. Z. Kopeliovich, ${ }^{2}$ C. B. Mezzetti, ${ }^{3}$ I. K. Potashnikova, ${ }^{2}$ and I. Schmidt ${ }^{2}$ \\ 1 Istituto Nazionale di Fisica Nucleare, Sezione di Perugia, \\ c/o Department of Physics, University of Perugia, Via A. Pascoli, I-06123, Italy \\ ${ }^{2}$ Departamento de Física, Universidad Técnica Federico Santa María and \\ Instituto de Estudios Avanzados en Ciencias e Ingenierí \\ Centro Científico-Tecnológico de Valparaíso; \\ Casilla 110-V, Valparaíso, Chile \\ ${ }^{3}$ Department of Physics, University of Perugia and Istituto Nazionale di Fisica Nucleare, \\ Sezione di Perugia, Via A. Pascoli, I-06123, Italy
}

(Dated: September 16, 2018)

\begin{abstract}
The so called number of hadron-nucleus collisions $n_{\text {coll }}(b)$ at impact parameter $b$, and its integral value $N_{\text {coll }}$, which are used to normalize the measured fractional cross section of a hard process, are calculated within the Glauber-Gribov theory including the effects of nucleon short- range correlations. The Gribov inelastic shadowing corrections are summed to all orders by employing the dipole representation. Numerical calculations are performed at the energies of the BNL Relativistic Heavy Ion Collider (RHIC) and CERN Large Hadron Collider (LHC). We found that whereas the Gribov corrections generally increase the value of $N_{\text {coll }}$, the inclusion of nucleon correlations, acting in the opposite directions, decreases it by a comparable amount. The interplay of the two effects varies with the value of the impact parameter.
\end{abstract}

PACS numbers: 24.85.+p, 13.85.Lg, 13.85.Lg, 25.55.Ci

\section{INTRODUCTION}

Much progress has been made recently improving the Glauber treatment 1] of high energy hadron-nucleus and nucleus-nucleus scattering. From one side, Gribov inelastic shadowing (IS) corrections [2] have been summed up to all orders within the light-cone dipole approach (see e.g. Refs. [3]-[5]). From the other side, the effects of short range correlations (SRC) on high-energy scattering has been revisited within realistic approaches [6]-[8]. In Ref. [1], the effects of Gribov IS and SRC on diffractive large rapidity gap (LRG) processes and total cross sections have been thoroughly analyzed. A particular motivation for precise calculations of these cross sections is the possibility to use them for normalization of other channels, which is sometimes a difficult task. Here such an analysis is extended to the calculation of the so-called number of collisions $n_{\text {coll }}(b)$ and its integral $N_{\text {coll }}$, two quantities that are used to normalize the fractional cross section of hard processes in proton-nucleus and nucleus-nucleus collisions. Our paper is organized as follows: in Sec III the basic elements necessary to treat SRC and Gribov IS in high-energy hadron-nucleus scattering are presented; the definition of the number of collision and their specific form within our approach, which goes beyond the Glauber model, are illustrated in Sec III, the Results of Calculations are presented in Sec IV and the summary and conclusions are given in Sec V

\section{GLAUBER APPROACH, GRIBOV INELASTIC SHADOWING AND SHORT RANGE CORRELATIONS}

As is well known, within the Glauber approach 1], the elastic hadron-nucleus amplitude reads as follows

$$
\Gamma^{h A}(\boldsymbol{b})=\left\{1-\left[1-\int \prod_{j=1}^{A} d^{3} r_{j} \Gamma_{j}^{h N}\left(\boldsymbol{b}-\boldsymbol{l}_{j}\right) \delta\left(\sum_{i=1}^{A} \boldsymbol{r}_{i}\right)\left|\psi_{0}\left(\boldsymbol{r}_{1} \ldots \boldsymbol{r}_{A}\right)\right|^{2}\right]\right\}
$$

where $\left\{\mathbf{r}_{i}\right\}=\left\{\mathbf{l}_{i}, z_{i}\right\}$ stands for a set of $(A-1)$ linearly independent intrinsic coordinates. This amplitude depends upon the modulus squared of the ground-state nuclear wave function $\left|\psi_{0}\left(\boldsymbol{r}_{1}, \ldots, \boldsymbol{r}_{A}\right)\right|^{2}$, which, as is now widely accepted, exhibits a complex correlation structure. Thus, the evaluation of various cross sections ac- counting for of all possible two-, three-, ..., many-body correlations, can be performed either by Monte Carlo many-fold numerical integration, or by using the following expansion of $\left|\psi_{0}\left(\boldsymbol{r}_{1}, \ldots, \boldsymbol{r}_{A}\right)\right|^{2}$ in terms of density matrices (see Refs. $[1,[9]$ ) 


$$
\left|\psi_{o}\left(\boldsymbol{r}_{1}, \ldots, \boldsymbol{r}_{A}\right)\right|^{2}=\prod_{j=1}^{A} \rho_{1}\left(\boldsymbol{r}_{j}\right)+\sum_{i<j} \Delta\left(\boldsymbol{r}_{i}, \boldsymbol{r}_{j}\right) \prod_{k \neq(i, j)} \rho_{1}\left(\boldsymbol{r}_{k}\right)+\sum_{(i<j) \neq(k<l)} \Delta\left(\boldsymbol{r}_{i}, \boldsymbol{r}_{j}\right) \Delta\left(\boldsymbol{r}_{k}, \boldsymbol{r}_{l}\right) \prod_{m \neq(i, j, k, l)} \rho_{1}\left(\boldsymbol{r}_{m}\right)+\ldots
$$

Here $\Delta\left(\boldsymbol{r}_{i}, \boldsymbol{r}_{j}\right)=\rho_{2}\left(\boldsymbol{r}_{i}, \boldsymbol{r}_{j}\right)-\rho_{1}\left(\boldsymbol{r}_{i}\right) \rho_{1}\left(\boldsymbol{r}_{j}\right)$ is the twobody contraction satisfying the sequential condition $\int d \boldsymbol{r}_{j} \Delta\left(\boldsymbol{r}_{i}, \boldsymbol{r}_{j}\right)=0$, and $\rho_{1}\left(\boldsymbol{r}_{i}\right)$ and $\rho_{2}\left(\boldsymbol{r}_{i}, \boldsymbol{r}_{j}\right)$ are the one- and two-body density matrices, normalized to 1 and obtained from the general equation $\rho_{n}\left(\boldsymbol{r}_{i}, \boldsymbol{r}_{2} \ldots \boldsymbol{r}_{n}\right)=$ $\int\left|\psi_{o}\left(\boldsymbol{r}_{1}, \boldsymbol{r}_{2}, \ldots, \boldsymbol{r}_{A}\right)\right|^{2} \prod_{n+1}^{A} d \boldsymbol{r}_{i}$. Note that in Eq. (2) the higher order terms, not explicitly displayed, include all possible products of unlinked two-body contractions, contributing to two-nucleon correlations, all possible products of unlinked three-body contractions, describing three-nucleon correlations, and so forth. Most Glauber-like calculations are based upon the singledensity approximation, consisting in disregarding all terms of the expansion (2) except the first one, i.e. $\left|\psi_{0}\left(\boldsymbol{r}_{1}, \ldots, \boldsymbol{r}_{A}\right)\right|^{2} \approx \prod_{j=1}^{A} \rho\left(\boldsymbol{r}_{j}\right)$. In the present paper, we go beyond such an approximation by considering higher order terms in Eq. (2), and will denote all quantities calculated within the single-density approximation by a superscript $g l$. Using the Glauber approach in the treatment of high-energy hadron-nucleus and nucleus-nucleus scattering is a common practice; however it should be stressed that in this way neither LRG processes nor intermediate diffractive hadron dissociation (Gribov IS) can be explicitly evaluated. It has been shown [3] that the light-cone dipole is an eigenstate of high-energy hadronnucleon interaction, and proper approaches have been developed to calculate LRG processes and Gribov IS to all orders within the single-density approximation (see, e.g., Refs [4]- 5]). Recently, the role played by the correlation terms in Eq. (2) has been revisited, showing [6] that twonucleon correlations (the effects of higher-order correlations being negligible) increase the total neutron-nucleus cross section at high energies, making the nucleus more opaque, in the opposite direction of Gribov IS which increases nuclear transparency. However in Ref. [6] Gribov IS has been considered only at the lowest order [10]. A coherent evaluation of IS and SRC on the total, $\sigma_{\text {tot }}^{h A}$, elastic, $\sigma_{e l}^{h A}$, quasi elastic, $\sigma_{q e l}^{h A}$, inelastic, $\sigma_{i n}^{h A}$, and diffractive dissociation hadron-nucleus inclusive cross sections, has been presented in Ref. 8], confirming the opposite roles played by SRC and IS. Following the formalism of Ref. [8], in the present paper we further analyze the effects of SRC and IS by considering inelastic hadron-nucleus collisions at high energies.

\section{NUMBER OF COLLISIONS $n_{\text {coll }}(b)$ AND $N_{\text {coll }}$}

\section{A. Glauber approach and short range correlations}

We are going to consider the normalization factor that is used to obtain the nucleus to nucleon ratio of cross sections of a hard reaction (high $p_{T}$, Drell-Yan, heavy flavor, etc), namely

$$
R_{A / N}^{h a r d}=\frac{\sigma_{\text {hard }}^{\text {hA }}}{A \sigma_{\text {hard }}^{\text {hN }}}
$$

The absolute value of a hard nuclear cross section is difficult to measure, and only the fraction of the total number of inelastic events $N_{\text {hard }}^{h A} / N_{\text {in }}^{h A}$ is known. One has therefore to normalize the fraction as follows

$$
R_{A / N}^{\text {hard }}=\frac{\sigma_{\text {in }}^{h A} N_{h a r d}^{h A}}{A \sigma_{\text {in }}^{h N} N_{h a r d}^{h N}}=\frac{1}{N_{\text {coll }}} \frac{N_{\text {hard }}^{h A}}{N_{\text {hard }}^{h N}},
$$

where

$$
N_{\text {coll }}=A \frac{\sigma_{i n}^{h N}}{\sigma_{i n}^{h A}}
$$

and $\sigma_{i n}^{h N}$ is the inelastic hadron-Nucleon inclusive cross section. Correspondingly, the number of hard collisions at a given impact parameter $b$ should be normalized as

$$
R_{A / N}^{\text {hard }}(b)=\frac{N_{\text {hard }}^{\text {hA }}(b)}{n_{\text {coll }}(b) N_{\text {hard }}^{h N}},
$$

where

$$
n_{\text {coll }}(b)=\frac{\sigma_{i n}^{h N} T_{A}(b)}{P_{i n}(b)},
$$

with $T_{A}(b)=\int d z \rho_{A}(|\mathbf{b}|, z)$ being the usual nuclear thickness function normalized to $A$. In Eq.(17) the numerator represents the number of possibilities of $h N$ interactions, and the denominator, $P_{i n}(b)$, is the probability for an inelastic interaction to occur at impact parameter $b$. Note that if the nuclear transparency decreases, $\sigma_{i n}^{h A}$ increases and $N_{\text {coll }}$ decreases, and viceversa. It has been stressed [4] that, when Eq. (5) is used to normalize hard data on $h A$ and $A A$, care must be taken in the definition of both the numerator and the denominator. The reasons are as follows. If the detector can detect all diffractive LRG processes, the numerator, which is taken from experimental data on $h N$ scattering, is $\sigma_{i n}^{h N}=\sigma_{t o t}^{h N}-\sigma_{e l}^{h N}$ and includes all diffractive channels; the denominator, which has to be evaluated 
within some models of hadron-nucleus scattering, is, accordingly, $\sigma_{i n}^{h A}=\sigma_{t o t}^{h A}-\sigma_{e l}^{h A}-\sigma_{q e l}^{h A}$ and includes all diffractive, LRG processes, except the so called quasi-elastic scattering, in which bound nucleons remain intact but the nucleus gets excited and decays to fragments. If the detector, on the contrary, cannot detect LRG processes, these have to be subtracted from the numerator and the denominator of Eq. (77). Our paper aims at analyzing various relevant models for the evaluation of the denominator. Let us start with the Glauber model and the single-density approximation, supposing that the detector cannot detect LRG processes. Within such a framework, $P_{i n}(b)$ is given by

$$
P_{i n}^{G l}(b)=1-e^{-\sigma_{i n}^{h N} T_{A}^{h}(b)},
$$

where $T_{A}^{h}(b)$ is the hadron-nucleus thickness function

$$
T_{A}^{h}(b)=\frac{2}{\sigma_{t o t}^{h N}} \int d^{2} l \operatorname{Re} \Gamma^{h N}(\boldsymbol{l}) T_{A}(\boldsymbol{b}-\boldsymbol{l}),
$$

with normalization $\int d^{2} b T_{A}^{h}(b)=A$, and the inclusive inelastic hadron-nucleus cross section is

$$
\sigma_{i n}^{h A}=\sigma_{t o t}^{h A}-\sigma_{e l}^{h A}-\sigma_{q e l}^{h A}=\int d^{2} b\left[1-e^{-\sigma_{i n}^{h N} T_{A}^{h}(b)}\right] .
$$

The total number of collisions will then read

$$
N_{\text {coll }}^{G l}=A \frac{\sigma_{i n}^{h N}}{\int d^{2} b\left[1-e^{-\sigma_{i n}^{h N} T_{A}^{h}(b)}\right]},
$$

where the denominator includes all diffractive LRG processes, but misses the effects from SRC and Gribov IS.
SRC can readily be implemented in Eq. (11) but the inclusion of Gribov IS is no easy theoretical task since the Glauber model, which is a single-channel approximation, cannot be applied, and more involved multichannel approach or the dipole representation should be used [4, 5, 8]. In this paper we have calculated $n_{\text {coll }}$, Eq. (7), and its integral $N_{\text {coll }}$, Eq. (5), with four different approaches for the evaluation of $\sigma_{i n}^{h A}$ : (i) the plain Glauber model within the single-density approximation given by Eq. (10); (ii) the improved Glauber model which includes the effects of SRC; and (iii) the model in which, besides SRC, also LRG processes and Gribov IS, calculated within the dipole approach, are included in $\sigma_{\text {tot }}^{h A}$, $\sigma_{e l}^{h A}$, and $\sigma_{q e l}^{h A} ;$ iv) finally, since frequently experiments select only events with particle production at central rapidities, missing LRG diffractive channels, we have modified accordingly [4] the model iii) by subtracting the cross sections of single and double diffraction from $\sigma_{i n}^{h N}$ in the numerators and the contribution of LRG processes from the denominator.

Let us briefly present the theoretical background underlying the above program (see Ref. [8] for more details). Concerning the effects of SRC on the various cross sections, it has been shown [6] that their inclusion is equivalent to the following modification of the hadron-nucleus thickness function,

$$
T_{A}^{h}(b) \Rightarrow \widetilde{T}_{A}^{h}(b)=T_{A}^{h}(b)-\Delta T_{A}^{h}(b)
$$

with the correlation correction $\Delta T_{A}^{h}(b)$ given by

$$
\Delta T_{A}^{h}(b)=\frac{1}{\sigma_{t o t}^{h N}} \int d^{2} l_{1} d^{2} l_{2} \Delta_{A}^{\perp}\left(\boldsymbol{l}_{1}, \boldsymbol{l}_{2}\right) \operatorname{Re} \Gamma^{p N}\left(\boldsymbol{b}-\boldsymbol{l}_{1}\right) \operatorname{Re} \Gamma^{p N}\left(\boldsymbol{b}-\boldsymbol{l}_{2}\right),
$$

where

$$
\Delta_{A}^{\perp}\left(\boldsymbol{l}_{1}, \boldsymbol{l}_{2}\right)=A^{2} \int_{-\infty}^{\infty} d z_{1} \int_{-\infty}^{\infty} d z_{2} \Delta\left(\boldsymbol{r}_{1}, \boldsymbol{r}_{2}\right)
$$

is the transverse two-nucleon contraction. In Eq. (13) two nucleon correlations, represented by all possible products of two-body contractions linked to all possible products of two $\Gamma^{p N}\left(\boldsymbol{b}-\boldsymbol{l}_{i}\right)$, are exactly summed up. It should be stressed that the inclusion of SRC does not affect the numerator of Eq. (11), but it will change the probability $P_{\text {in }}(b)$, which is still given by Eq. (8) but with $T_{A}^{h}(b)$ Eq. (9) replaced by $\widetilde{T}_{A}^{h}(b)$ Eq. (12).

\section{B. Adding Gribov corrections via light-cone dipoles}

The effects of both Gribov IS and SRC have been considered as in Refs. [4, 5, 8] within the light-cone dipole approach. The dipole representation for the amplitude of hadronic interactions allows one to sum up the Gribov inelastic corrections to all orders. If the collision energy is high enough to keep the dipole size "frozen" by Lorentz time delation during propagation through the nucleus, the calculations are much simplified. The key ingredients of the approach are the universal dipole-nucleon cross section $\sigma_{d i p}\left(r_{T}, s\right)\left(r_{T}\right.$ is the transverse dimension of the $\bar{q} q$ dipole and $s$ is the energy) and the light-cone wave function of the projectile hadron $\Psi_{N}\left(\boldsymbol{r}_{1}, \boldsymbol{r}_{2}, \boldsymbol{r}_{3}\right)[5]$. 
In this paper, as in Ref. [8], the models for both quantities have been taken from Ref. [5]. Both models employ the saturated shape of the dipole cross section and differ only by modeling the proton wave function. For the sake of illustration, let us consider $\sigma_{t o t}^{p A}$. Taking into account both Gribov IS and SRC, one gets [8]

$$
\sigma_{\text {tot }}^{p A}=2 \int d^{2} b\left[1-\left\langle e^{-\frac{1}{2} \sigma_{d i p}\left(r_{T}, s\right) \widetilde{T}_{A}^{d i p}\left(b, r_{T}, \alpha\right)}\right\rangle\right]
$$

where the average is over the transverse size of the dipole $q \bar{q}$ and the fractional light-cone momentum $\alpha$, i.e., for a generic function $f\left(r_{T}, \alpha\right)$,

$$
\left\langle f\left(r_{T}, \alpha\right)\right\rangle \equiv \int_{0}^{1} d \alpha \int_{0}^{\infty} d^{2} r_{T}\left|\Psi_{N}\left(r_{T}, \alpha\right)\right|^{2} f\left(r_{T}, \alpha\right) .
$$

The correlated dipole tickness function reads

$$
\widetilde{T}_{A}^{d i p}\left(b, r_{T}, \alpha\right)=T_{A}^{d i p}\left(b, r_{T}, \alpha\right)+\Delta T_{A}^{d i p}\left(b, r_{T}, \alpha\right)
$$

with

$$
\begin{gathered}
T_{A}^{d i p}\left(b, r_{T}, \alpha\right)= \\
=\frac{2}{\sigma_{d i p}\left(r_{T}\right)} \int d^{2} l \operatorname{Re} \Gamma^{d i p}\left(\boldsymbol{l}, \boldsymbol{r}_{T}, \alpha\right) T_{A}(\boldsymbol{b}-\boldsymbol{l})
\end{gathered}
$$

and

$$
\Delta T_{A}^{d i p}\left(b, r_{T}, \alpha\right)=\frac{1}{\sigma_{d i p}\left(r_{T}\right)} \int d^{2} l_{1} d^{2} l_{2} \Delta_{A}^{\perp}\left(\boldsymbol{l}_{1}, \boldsymbol{l}_{2}\right) \operatorname{Re} \Gamma^{d i p}\left(\boldsymbol{b}-\boldsymbol{l}_{1}, r_{T}, \alpha\right) \operatorname{Re} \Gamma^{d i p}\left(\boldsymbol{b}-\boldsymbol{l}_{2}, r_{T}, \alpha\right) .
$$

Here $\Delta_{A}^{\perp}\left(\boldsymbol{l}_{1}, \boldsymbol{l}_{2}\right)$ is given by Eq. (14) and the partial dipole-nucleon amplitude $\operatorname{Re} \Gamma^{d i p}$ is given by Eq. (41) of Ref. [8]. Using the above equations, the total cross section takes the form,

$$
\sigma_{\text {tot }}^{p A}=2 \int d^{2} b\left\{1-e^{\frac{1}{2} I_{A}(b)}\left\langle e^{-\frac{1}{2} \sigma_{d i p} T_{A}^{h}(b)}\right\rangle\right\}
$$

where the quantity $I_{A}(b)$ contains the effects from SRC [8].

\section{RESULTS OF CALCULATIONS}

We have calculated $n_{\text {coll }}$ and $N_{\text {coll }}$ in proton-nucleus scattering at BNL Relativistic Heavy Ion Collider (RHIC) and CERN Large Hadron Collider (LHC)LHC energies within the following approaches: i) the Glauber model with single-density approximation

$$
n_{c o l l}^{G l}(b)=\frac{\sigma_{i n}^{h N} T_{A}(b)}{1-e^{-\sigma_{i n}^{h N} T_{A}^{h}(b)}}
$$

ii) the Glauber model plus SRC

$$
n_{\text {coll }}^{G l+S R C}(b)=\frac{\sigma_{i n}^{h N} T_{A}(b)}{1-e^{-\sigma_{i n}^{h N}} \widetilde{T}_{A}^{h}(b)} ;
$$

iii) the Glauber model plus SRC and Gribov IS corrections, using the results of Ref. [8]. In this case, we have also considered the possibility that the detector misses LRG processes which, therefore, have to be subtracted from the numerator and the denominator of Eq. (22), arriving at

$$
n_{\text {coll }}^{G l+S R C+I S}(b)=\frac{\left(\sigma_{i n}^{h N}-\sigma_{d i f f}^{h N}\right) T_{A}(b)}{P_{i n}(b)},
$$

where the probability $P_{i n}(b)$ is given by

$$
P_{i n}(b)=\frac{d \sigma_{t o t}^{h A}}{d^{2} b}-\frac{d \sigma_{e l}^{h A}}{d^{2} b}-\frac{d \sigma_{d i f f}^{h A}}{d^{2} b}-\frac{d \sigma_{q e l}^{h A}}{d^{2} b}-\frac{d \sigma_{q s d}^{h A}}{d^{2} b},
$$

with

$$
\begin{gathered}
\frac{1}{2} \frac{d \sigma_{\text {tot }}^{h A}}{d^{2} b}=1-e^{\frac{1}{2} I_{A}(b)}\left\langle e^{-\frac{1}{2} \sigma_{d i p} T_{A}^{h}(b)}\right\rangle \\
\frac{d\left(\sigma_{e l}^{h A}+\sigma_{d i f f}^{h A}\right)}{d^{2} b}=\frac{d \sigma_{t o t}^{h A}}{d^{2} b}-1+e^{I_{A}(b)}\left\langle e^{-\sigma_{d i p} T_{A}^{h}(b)}\right\rangle, \\
\frac{d\left(\sigma_{q e l}^{h A}+\sigma_{q s d}^{h A}\right)}{d^{2} b}= \\
\left\langle e^{-\sigma_{d i p} T_{A}^{h}(b)}\left\{e^{\tilde{I}_{A}(b)} e^{\frac{\sigma_{d i p}^{2} T_{A}^{h}(b)}{16 \pi B_{e l}}}-e^{I_{A}(b)}\right\}\right\rangle
\end{gathered}
$$

Here the quantities $I_{A}(b)$ and $\tilde{I}_{A}(b)$, providing the effects from SRC are 
$p-{ }^{208} \mathrm{~Pb}$

GLAUBER

\begin{tabular}{c|cccccc}
\hline \hline & $\sigma_{i n}^{p N}[m b]$ & $\sigma_{\text {tot }}^{p A}[m b]$ & $\sigma_{e l}^{p A}[m b]$ & $\sigma_{q e l}^{p A}[m b]$ & $\sigma_{\text {in }}^{p A}[m b]$ & $N_{\text {coll }}$ \\
\hline RHIC & 42.1 & 3297.6 & 1368.4 & 66.0 & 1863.2 & 4.70 \\
\hline LHC & 68.3 & 3850.6 & 1664.8 & 121.0 & 2064.8 & 6.88 \\
\hline \hline
\end{tabular}

GLAUBER+SRC

\begin{tabular}{c|cccccc}
\hline \hline & $\sigma_{\text {in }}^{p N}[\mathrm{mb}]$ & $\sigma_{\text {tot }}^{p A}[\mathrm{mb}]$ & $\sigma_{\text {el }}^{p A}[\mathrm{mb}]$ & $\sigma_{q e l}^{p A}[\mathrm{mb}]$ & $\sigma_{\text {in }}^{p A}[\mathrm{mb}]$ & $N_{\text {coll }}$ \\
\hline $\mathrm{RHIC}$ & 42.1 & 3337.6 & 1398.1 & 58.5 & 1881.0 & 4.65 \\
\hline LHC & 68.3 & 3885.8 & 1690.5 & 112.6 & 2082.7 & 6.82 \\
\hline \hline
\end{tabular}

GLAUBER+SRC+GRIBOV IS

\begin{tabular}{c|cccccc}
\hline \hline & $\sigma_{i n}^{p N}\left(\sigma_{i n}^{p N}-\sigma_{d i f f}^{p N}\right)[m b]$ & $\sigma_{\text {tot }}^{p A}[m b]$ & $\sigma_{e l}^{p A}\left(\sigma_{e l}^{p A}+\sigma_{\text {diff }}^{p A}\right)[m b]$ & $\sigma_{q e l}^{p A}\left(\sigma_{q e l}^{p A}+\sigma_{q s d}^{p A}\right)[m b]$ & $\sigma_{i n}^{p A}[m b]$ & $N_{c o l l}$ \\
\hline RHIC & $42.1(30.0)$ & 3228.1 & $1314.0(1331.0)$ & $72.0(74.4)$ & $1842.1(1823.0)$ & $4.75(3.42)$ \\
\hline LHC & $68.3(56.3)$ & 3833.3 & $1655.7(1658.0)$ & $113.4(111.3)$ & $2064.2(2064.0)$ & $6.88(5.67)$ \\
\hline \hline
\end{tabular}

TABLE I: $N_{\text {coll }}$ Eq. (5) in $p-{ }^{208} \mathrm{~Pb}$ scattering at RHIC and LHC energies calculated by integrating the numerator and denominator of Eqs. (21), (22), and (23). In the latter case (GLAUBER+SRC+GRIBOV)the values in parentheses correspond to to the full Eqs. (23)- (27) and represent the case when the detector misses LRG events, whereas the other values have been obtained by omitting the diffractive cross section $\sigma_{d i f f}^{h N}$ from Eq. (23) and $d \sigma_{d i f f}^{h A} / d^{2} b$ and $d \sigma_{q s d}^{h A} / d^{2} b$ from Eq. (24), to describe processes when LRG events are detected.

$$
I_{A}(b)=\left\langle\sigma_{\bar{q} q}\left(r_{T}\right) \Delta T_{A}^{\bar{q} q}\left(b, r_{T}, \alpha\right)\right\rangle=\left[\sigma_{e l}^{p N}+\sigma_{s d}^{p N}\right] \times \int d^{2} \delta \exp \left[-\frac{\delta^{2}}{4 B(s)+R_{0}^{2}(s) / 2}\right] \Delta_{A}^{\perp}(\vec{\delta}, b)
$$

and

$$
\tilde{I}_{A}(b) \approx\left[\frac{\sigma_{t o t}^{p N}-\sigma_{e l}^{p N}-\sigma_{s d}^{p N}}{\sigma_{t o t}^{p N}}\right]^{2} I_{A}(b)
$$

In these equations, $\sigma_{\text {diff }}^{h N}$ includes the diffraction dissociation of the projectile and target hadrons, $\sigma_{\text {diff }}^{h A}$ includes the diffraction dissociation of the projectile $\left(\sigma_{s d}\right.$ of Ref. [8]), and $\sigma_{q s d}^{h A}$ the diffraction dissociation of both the projectile hadron and the target nucleus (see Ref. [8] for more details and notations). Equations (23)-(27) have been calculated considering and omitting the diffractive cross sections $\sigma_{\text {diff }}^{h N}, \sigma_{\text {diff }}^{h A}$ and $\sigma_{q s d}^{h A}$; the former case corresponds to a probability $P_{i n}(b)$, which, when integrated, yields the non diffractive cross section and represents the case when the detector is insensitive to LRG channels (e.g. in the experiments STAR [11], PHENIX [12] and PHOBOS [13] at RHIC); the second case corresponds to the assumption that the detector can detect the LRG processes. In the calculations we used the experimental cross sections $\sigma_{i n}^{N N}=42 m b$ and $\sigma_{i n}^{N N}=68.43 m b$ for RHIC and LHC energies, respectively. As for the nuclear quantities, realistic one- and two- body densities and correla- tion functions from Ref. [7] have been adopted (see Ref. 8] for details). Notice that, eventually, by definition, the nuclear thickness function $T_{A}(b)$ has to be used in the numerators of Eqs. (21)-(24) since, as already pointed out, the number of opportunities to perform a hard collision depends only upon the single-particle density; the denominator, on the contrary, is affected by SRC, since these couple two-body contractions with products of two profile $\Gamma$, according to Eqs. (12) and (19). The results of calculations pertaining to $p-{ }^{208} \mathrm{~Pb}$ collisions are presented in Table I. It can be seen that SRC increase the value of $\sigma_{i n}^{N A}$ and, correspondingly, decrease the values of $N_{\text {coll }}$; at the same time, Gribov IS increases them back to values near to the Glauber ones. Similar results are obtained for other nuclei. The effects of both SRC and Gribov IS amounts to a few percent, in agreement with the results of the calculation of deuteron-gold scattering [4]. Our results, together with the results previously 
obtained for LRG cross sections, generally show that, although the separate effects of Gribov IS and SRC on the quantities we have considered can be appreciable, they tend to act in the opposite directions, making the final result similar to the one obtained within the Glauber approximation. However, it should also be pointed out that when the detector misses the LRG processes (the values in parentheses), $N_{\text {coll }}$ turns out to be appreciably lower than the Glauber value.

\section{SUMMARY AND CONCLUSIONS}

In this paper, we continued our analysis of the effects of short-range correlations and Gribov inelastic shad- owing on high-energy scattering processes by considering the number of collisions, the quantity that is used to normalize the fractional cross section of hard processes in proton-nucleus and nucleus-nucleus collisions. The short-range correlations are treated within a realistic many-body framework, and the effects of Gribov inelastic shadowing are summed up to all orders by the lightcone dipole approach. The numerical results confirm the opposite role played by correlations and Gribov inelastic shadowing and, once again, point at the necessity of inclusion of both effects whenever a precise analysis of experimental data is required.
[1] R. J. Glauber, in Lectures in Theoretical Physics, W. E. Brittin et al Editors, New York (1959).

[2] V. N. Gribov, Sov. Phys. JETP 29, 483 (1969); Zh. Eksp. Teor. Fiz. 56, 892 (1969).

[3] B. Z. Kopeliovich, L. I. Lapidus and A. B. Zamolodchikov, JETP Lett. 33, 595 (1981) [Pisma Zh. Eksp. Teor. Fiz. 33, 612 (1981)].

[4] B. Z. Kopeliovich, Phys. Rev. C68 (2003) 044906.

[5] B. Z. Kopeliovich, I. K. Potashnikova and I. Schmidt, Phys. Rev. C73 (2006) 034901

[6] M. Alvioli, C. Ciofi degli Atti, I. Marchino, V. Palli and H. Morita, Phys. Rev. C78 (2008) 031601(R).

[7] M. Alvioli, C. Ciofi degli Atti, and H. Morita, Phys. Rev. Lett. 100 (2008) 162503.

[8] M. Alvioli, C. Ciofi degli Atti, B. Z. Kopeliovich, I.
K. Potashnikova and I. Schmidt, Phys. Rev. C81 (2010) 025204.

[9] L. L. Foldy, J. D. Walecka, Ann. Phys. (NY) 54 (1969) 447.

[10] V. A. Karmanov, L. A. Kondratyuk, JETP Lett. 18 (1973) 162503.

[11] J. Adams et al (STAR Collaboration), Phys. Rev. Lett. 91 (2003) 172302. ArXiv: nucl-ex/0305015.

[12] K. Adcox et al (PHENIX Collaboration), Phys.Rev.Lett. 91 (2003) 072303. ArXiv: nucl-ex/0306021.

[13] B.B. Back et al (PHOBOS Collaboration), Phys.Rev.Lett. $91 \quad$ (2003) 072302. ArXiv: nucl-ex/0306025. 